\title{
Erratum
}

\section{Erratum for "Higher-Order Weakly Generalized Adjacent Epiderivatives and Applications to Duality of Set-Valued Optimization"}

\author{
Qi-Lin Wang \\ College of Sciences, Chongqing Jiaotong University, Chongqing 400074, China \\ Correspondence should be addressed to Qi-Lin Wang, wangq197@126.com \\ Received 24 September 2010; Accepted 27 January 2011 \\ Copyright (C) 2011 Qi-Lin Wang. This is an open access article distributed under the Creative \\ Commons Attribution License, which permits unrestricted use, distribution, and reproduction in \\ any medium, provided the original work is properly cited.
}

An important property is established for higher-order weakly generalized adjacent epiderivatives. This corrects an earlier result by Wang and Li (2009).

\section{Introduction}

The concept of higher-order weakly generalized adjacent epiderivatives is introduced, and an important property is given for the derivatives in [1].

Proposition 1.1. Let $E$ be a nonempty convex subset of $X, x, x_{0} \in E, y_{0} \in F\left(x_{0}\right)$. Let $F-y_{0}$ is $C$-convexlike on $E, u_{i} \in E, v_{i} \in F\left(u_{i}\right)+C, i=1,2, \ldots, m-1$. If the set $q\left(x-x_{0}\right):=\{y \in Y \mid$ $\left.\left(x-x_{0}, y\right) \in G-T_{\mathrm{epi}(F)}^{b(m)}\left(x_{0}, y_{0}, u_{1}-x_{0}, v_{1}-y_{0}, \ldots, u_{m-1}-x_{0}, v_{m-1}-y_{0}\right)\right\}$ fulfills the weak domination property for all $x \in E$, then

$$
F(x)-y_{0} \subset d_{w}^{b(m)} F\left(x_{0}, y_{0}, u_{1}-x_{0}, v_{1}-y_{0}, \ldots, u_{m-1}-x_{0}, v_{m-1}-y_{0}\right)\left(x-x_{0}\right)+C
$$

For other notations and definitions, one may refer to [1].

While proving Proposition 1.1 in [1], the authors used the assumption that the $F-y_{0}$ is $C$-convexlike (see $[2,3])$ on a convex set $E$ which implies cone $\left(e p i(F)-\left(x_{0}, y_{0}\right)\right)$ is a convex cone. In fact, the assumption may not hold. The following example shows that the case and Proposition 1.1 may not hold, where one only takes $m=2$. 
Example 1.2. Let $X=Y=R, C=R_{+}, E=[-1,2] \subset R$. Consider a set-valued map $F: E \rightarrow 2^{Y}$ defined by

$$
F(x)= \begin{cases}\{y \in Y \mid y \geq 0\}, & \text { if } x \in(-1,2], \\ \{-1\}, & \text { if } x=-1 .\end{cases}
$$

Take $\left(x_{0}, y_{0}\right)=(0,0) \in \operatorname{graph}(F), u=1, v=0 \in F(1)+C$. Naturally, $F-y_{0}$ be $C$-convexlike on $E$, but cone $\left(\right.$ epi $\left.(F)-\left(x_{0}, y_{0}\right)\right)$ is not a convex cone.

On the other hand, for any $x \in E, q\left(x-x_{0}\right):=\left\{y \in Y \mid\left(x-x_{0}, y\right) \in G-T_{\mathrm{epi}(F)}^{b(2)}\left(x_{0}, y_{0}, u-\right.\right.$ $\left.\left.x_{0}, v-y_{0}\right)\right\}=C$ fulfills the weak domination property. Thus, the assumptions of Proposition 1.1 are satisfied. But, for $x=-1 \in E$,

$$
\begin{gathered}
F(-1)-y_{0}=\{-1\}, \\
d_{w}^{b(m)} F\left(x_{0}, y_{0}, u-x_{0}, v-y_{0}\right)\left(-1-x_{0}\right)+C=C,
\end{gathered}
$$

which shows that the inclusion of (1.1) does not hold here.

\section{Properties of Higher-Order Weakly Generalized Adjacent Epiderivatives}

In this section, one presents an important property of higher-order weakly generalized adjacent epiderivatives which is a correction of [1, Proposition 3.14]. Firstly, one gives a notation of generalized cone-convex set-valued maps.

Definition 2.1. Let $F: E \rightarrow 2^{\gamma}$ be a set-valued map, $x_{0} \in E,\left(x_{0}, y_{0}\right) \in \operatorname{graph}(F)$. $F$ is said to be generalized $C$-convex at $\left(x_{0}, y_{0}\right)$ on $E$, if cone(epi $\left.F-\left(x_{0}, y_{0}\right)\right)$ is convex.

Remark 2.2. If $F$ is $C$-convex on convex set $E$ (see [4]), then, $F$ is generalized $C$-convex at $\left(x_{0}, y_{0}\right) \in \operatorname{graph}(F)$ on $E$. But the converse may not hold. The following example shows the case.

Example 2.3. Let $E=[-1,1] \subset R, C=R_{+}$. Consider a set-valued map $F: E \rightarrow 2^{R}$ defined by

$$
F(x)=\left\{y \in R \mid y \geq x^{2 / 3}\right\}, \quad \forall x \in E
$$

Take $\left(x_{0}, y_{0}\right)=(0,0) \in \operatorname{graph}(F)$. Then $F$ is generalized $C$-convex at $\left(x_{0}, y_{0}\right)$ on $E$, but $F$ is not $C$-convex on $E$. 
Proposition 2.4. Let E be a nonempty convex subset of $X, x, x_{0} \in E, y_{0} \in F\left(x_{0}\right)$. Let $F$ be generalized $C$-convex at $\left(x_{0}, y_{0}\right)$ on $E, u_{i} \in E, v_{i} \in F\left(u_{i}\right)+C, i=1,2, \ldots, m-1$. If the set $q\left(x-x_{0}\right):=\{y \in$ $\left.Y \mid\left(x-x_{0}, y\right) \in G-T_{\text {epi }(F)}^{b(m)}\left(x_{0}, y_{0}, u_{1}-x_{0}, v_{1}-y_{0}, \ldots, u_{m-1}-x_{0}, v_{m-1}-y_{0}\right)\right\}$ fulfills the weak domination property for all $x \in E$, then, for any $x \in E$, one obtains

$$
F(x)-y_{0} \subset d_{w}^{b(m)} F\left(x_{0}, y_{0}, u_{1}-x_{0}, v_{1}-y_{0}, \ldots, u_{m-1}-x_{0}, v_{m-1}-y_{0}\right)\left(x-x_{0}\right)+C
$$

Proof. The proof follows on the lines of [1, Proposition 3.14] by using generalized C-convex instead of $C$-convexlike.

Remark 2.5. In [1, Remark 3.15], one should use "generalized cone-convex" instead of "coneconvexlikeness". In $[1$, Theorems $4.5,4.7,5.2]$, one should use " $(F, G)$ is generalized $C \times D$ convex at $\left(x_{0}, y_{0}, z_{0}\right)$ on a nonempty subset $E^{\prime \prime}$ instead of " $(F, G)$ is $C \times D$-convexlike on a nonempty convex subset $E^{\prime \prime}$.

\section{Acknowledgments}

The author would like to thank Professor S. J. Li and the anonymous referees for valuable comments and suggestions. This paper was partially supported by Science and Technology Research Project of Chong Qing Municipal Education Commission (KJ100419), the National Natural Science Foundation of China (no. 10871216 and no. 11071267), the Natural Science Foundation Project of CQ CSTC, and the Excellent Young Teachers Program of Chongqing Jiaotong University, China.

\section{References}

[1] Q. L. Wang and S. J. Li, "Higher-order weakly generalized adjacent epiderivatives and applications to duality of set-valued optimization," Journal of Inequalities and Applications, vol. 2009, Article ID 462637, 18 pages, 2009.

[2] Z.-F. Li and G.-Y. Chen, "Lagrangian multipliers, saddle points, and duality in vector optimization of set-valued maps," Journal of Mathematical Analysis and Applications, vol. 215, no. 2, pp. 297-316, 1997.

[3] G. Y. Chen and W. D. Rong, "Characterizations of the Benson proper efficiency for nonconvex vector optimization," Journal of Optimization Theory and Applications, vol. 98, no. 2, pp. 365-384, 1998.

[4] H. W. Corley, "Existence and Lagrangian duality for maximizations of set-valued functions," Journal of Optimization Theory and Applications, vol. 54, no. 3, pp. 489-501, 1987. 